\title{
The scale development of new citizens' adaptation to urban society in the process of China's urbanization
}

\author{
Kai Du ${ }^{1}$, Xiaofu Pan ${ }^{1,2, *}$ \\ ${ }^{1}$ School of Culture \& Social Development Studies, Southwest University, Chongqing, China \\ ${ }^{2}$ Research Center of Psychology and Social Development, Southwest University, Chongqing, China
}

Email address:

smilepxf@126.com (Xiaofu Pan),dukai0929@126.com (Kai Du)

To cite this article:

Kai Du, Xiaofu Pan. The Scale Development of New Citizens' Adaptation to Urban Society in the Process of China's Urbanization. Psychology and Behavioral Sciences. Vol. 3, No. 3, 2014, pp. 93-99. doi: 10.11648/j.pbs.20140303.12

\begin{abstract}
Urbanization expedites numerous new citizens and a set of effective assessment tools are needed to evaluate their adaptation to urban society. This study attempts to develop a scale in Chinese context. The theoretical framework of scale is constructed through open questionnaire surveys and in-depth interviews, and the items of scale is developed on the basis of theoretical structure. By the exploratory factor analysis of pretesting data, the results show the scale is made up of 43 items, and including five dimensions: work adaptation, environmental adaptation, interpersonal adaptation, values for adaptation and psychological adaptation. Then the five factors mode are verified by confirmatory factor analysis of 580 data testing formally. The results indicate that the scale has a good construct validity and reliability. It can serve as the tool to evaluate new citizens' adaptation to urban society.
\end{abstract}

Keywords: Urbanization, New Citizens, Adaptation to Urban Society, Scale Development

\section{Introduction}

China's urbanization has developed rapidly since Reform and Opening-up. In 2012, 145.7 million inhabitants transformed their rural household registration systems into urban ones compared with that of 1990. Furthermore, 0.2 billion agricultural population will become urban population in next two decades. These new citizens are apt to bother about adaptation to urban society because they have to experience the transformation of space, mode of thinking and psychological consciousness, etc. Social adaptation is the basic quality of new citizens who are expected to change life style, the way of thinking and doing in order to integrate themselves into urban life. According to some researches, social adaptation is an important criterion to evaluate mental health [1] and poor social adaptation lead to externalizing and internalizing behavioral problems and make them integrate into city life difficultly [2]. Besides, poor social adaptation has significant relationship with criminal behavior [3]. Hence, improving new citizens' adaptation to urban society is of great significance to individuals, the whole country even society.

Social adaptation is always a hot topic among western scholars. The researchers study it from the perspective of biology, sociology, psychology, anthropology, etc. and achieve a lot [4]. For instance, western sociologists put forward "assimilation theory" and "multiculturalism" about immigrants' adaptation to society [5]. Cain, Levine \& Elzey (1963) developed Cain-Levine Social Competency Scale; Nihira, Foster, Shellhaas \& Leland (1975) developed Social Behavior Scale; Mercer \& Lewis (1978)developed Adaptive Behavior Inventory for Children [6]. The vast majority of studies on social adaptation focus on children, teenagers, college students and some special groups [7].For example, Wang \&Lin, et al. [8] developed the Scale of Children's Adaptation to Social Life. Yang \& Jin [9] developed the Middle School Student's Social Adaptation Scale. Fang, Wo\& Lin [10] developed Chinese College Students' Adaptation Scale. Xiao\& Miao, et al. [11] developed the Assessment Scale of Recruits' Adaptation to Military Life. Some of the aforementioned scales are developed for the general public, some for children, teenagers, college students and troops. However, when those scales are used to measure urban social adaptation of new citizens, it lacks of accuracy and validity. Meanwhile, accurate and scientific assessment and judgment are necessary for the improvement of new citizens' adaptation to urban society. Therefore, the development of a set of assessment tools is of practical and profound significance for the scientific grasp and promotion of new citizens' smooth adaptation to urban society. 
Different people have different opinions on the definition of social adaptation in the field of sociological and psychological study. From the perspective of individuals' relations with society, Leland (1973) and Cone (1987) think that social adaptation is individual's reaction to social culture, values and the way of life when interacting with society [12]. According to Zhu (1989), social adaptation is the process of individuals' acceptance of existing ways of social life, morals \& ethics and code of behaviors [13]. In terms of personal capabilities, Paris \& White-Williams (2005) define social adaptation as the fulfillment of working $\&$ social roles and responsibilities [14]. Taking the levels of personality into consideration, $\mathrm{Lu}$ (2003) holds the view that social adaptation is people's adaptation to social surroundings on the basis of the levels of personality and it's peculiar to humans [15]. From social perspective, social adaptation consists of the following parts: First aspect is adaptation to social environment, which appropriate transition of different conditions and ways of life is included Second aspect is adaptation to various social roles, which covers the proper transformation of sundry awareness of roles and codes of conduct. Third aspect is adaptation to social activities, which involves corresponding changes of various activity rules and abilities [16].The aforesaid research discusses and defines social adaptation from the perspective of individual's relations with society, individual capabilities, levels of personality and socialization respectively. Yet, further studies are urgent owing to the lack of construction of dimensions scale and empirical analysis.

From the perspective of cultural learning, social adaptation includes acculturation attitudes, conational cultural maintenance, host acculturative experience and language competency [17]. Berry \& Phinney et al.'s study of young immigrants' psychological adaptation and adaptation to social culture involves five aspects: life satisfaction, self-esteem, psychological problem, school adjustment and behavior problem [18]. Jasinskajap-Lahti (2008) divides immigrant adaptation into three dimensions (psychological adaptation, social culture adaptation and social economics adaptation) when investigating immigrants of former Soviet Union [19]. Tao (2000) classifies the operation of college students' enrollment adaptation into five dimensions: learning adaptation, interpersonal adaptation, selfdependence, environmental identity and physical \& mental manifestation [20]. Tian (1995) categorizes rural migrant workers' social adaptation as economic adaptability, social adaptability and psychological adaptability [21]. Based on a survey conducted by Feng, social adaptation of rural migrants of the Three Gorges is divided into four dimensions: psychological adaptation, economic adaptation, environmental adaptation and life adaptation [22]. Besides, Wang et al. (2013) classify new citizens' adaptation to city life as adaptation to living conditions, adaptation to city environment, interpersonal adaptation and adaptation to working situation [23].

By synthesizing the different theories above, this study holds that new citizens' adaptation to urban society refers to appropriately transform their awareness of life and economic environment, interpersonal relations and cultural conception as well as the inner sense of belonging according to the changes of environment. It contains five aspects. First aspect is work adaptation, which involves the individual's capability of dealing with city jobs and coordinating incomes. Second aspect is environmental adaptation, which means that individual is able to change themselves to adapt to the physical environment and surroundings properly. Third aspect is interpersonal adaptation, which talks about individual's ability to establish harmonious relationship with original inhabitants by changing traditional ways of communication. Fourth aspect is values for adaptation, which concerns individual's capability of adapting to the mainstream of cultural conception and ways of thinking through appropriate transformation. Fifth aspect is psychological adaptation, which refers to individual can coordinate various mental feelings when interacting with subjective and objective environment. In terms of the operational definition and theoretical construction of new citizens' adaptation to urban society, this study develops relevant scale and verifies its reliability and validity.

\section{Methods}

\subsection{Development of Formal Questionnaire}

\subsubsection{Collecting Data}

In terms of the operational definition of adaptation to urban society, we regard new citizen's self-perception and adaptive state as theoretical assumption and develop open-ended questionnaires. The questions are designed as follows: (1) What do you think of new citizens' adaptation to urban society? (Issues like living \& working environment, cultural conception, inner feeling etc. should be considered.) (2) Please evaluate your own adaptation to urban society and explain in detail what you can adapt to and what you can't. (3) Which factors exert considerable influences on your adaptation to urban society? (4) What will you do in order to improve new citizens' capability for adaptation to urban society? The opened questionnaire was conducted among 193 new citizens from 17 communities of 9 different districts in Chongqing, respectively. The total number of valid return was 178 (male $\mathrm{N}=101$, female $\mathrm{N}=86$ ). Meanwhile, we conducted in-depth interviews with 76 new citizens among them (male $\mathrm{N}=42$, female $\mathrm{N}=34$ ).

\subsection{Developing Item}

The primary scale of 60 items was developed through classifying and concluding 187 open-ended questionnaires and 76 transcripts of in-depth interviews. Participants were asked to respond to each item using a 5 point Likert scale ( $1=$ strongly disagree, $5=$ strongly agree $)$.

\subsection{Participants}

Pretest: 450 participants (male $\mathrm{N}=236$, female $\mathrm{N}=203$ ) were randomly chosen from 60 communities of 17 different 
districts in Chongqing. 61 are poor. 311 can support themselves basically. 67 belong to the rich. The 439 valid copies got recovered from 450 questionnaires with returns-ratio $97.5 \%$.

Retest: According to three levels of the community (large, medium and small), 603 participants (male $\mathrm{N}=319$, female $\mathrm{N}=261$ ) were chosen by stratified-random sampling method from 72 communities of 25 different districts in Chongqing. 74 are poor, 420 only have adequate food and clothing, and 86 are rich. The 580 valid copies got recovered from 603 questionnaires with returns-ratio $96.1 \%$.

\subsection{Procedure}

With uniform instruction, highly trained personnel ask participants to answer the anonymous questionnaire. Then all data would be analyzed using statistical software SPSS 20.0 and LISREL8.70.

\section{Results}

\subsection{Exploratory Factor Analysis}

According to the results of principal component analysis and rotated by Varimax, we removed items of low loading $(\mathrm{r}<0.4)$ and double loading (the difference between the two factors loading $r<0.4)$. Then the formal questionnaire that includes 43 items is formed. Based on scree plot test, it is reasonable to extract five factors, which explain $47.8 \%$ of the total variance. Results of exploratory factor analysis are shown from table 1 to table 5 .

Table 1. Work adaptation

\begin{tabular}{llc}
\hline Variable & Item & Factor Loading \\
\hline VAR00032 & I can't find a job for the higher techniques or abilities of job requirements. & .634 \\
VAR00028 & There are much potential safety hazard of my work environment. & .584 \\
VAR00030 & The current work environment makes me sad. & .561 \\
VAR00026 & We are often engaged in low-paid works which are dirty, heavy and tired. & .546 \\
VAR00035 & I am disappointed at the payment in cities. & .521 \\
VAR00031 & It has higher requirements for abilities and techniques of work after changing permanent resident registration. & .484 \\
VAR00013 & It is hard for me to accept the nervous and fast-paced life in cities. & .483 \\
VAR00019 & It is hard for me to accept the consumption level of my living city. & .453 \\
VAR00014 & It always makes me extremely tired of the timetable in cities. & .449 \\
Eigenvalue & 3.49 & \\
Cumulative $\%$ & 8.12 & \\
\hline
\end{tabular}

Table 2. Environment adaptation

\begin{tabular}{lll}
\hline Variable & Item & Factor Loading \\
\hline VAR00023 & The weather variations of my living place make me ill. & .773 \\
VAR00016 & It's hard for me to accept the dietary habit of city. & .747 \\
VAR00022 & It's difficult for me to adapt the climate of my living place. & .734 \\
VAR00024 & I feel ill for the climate of my current living place. & .723 \\
VAR00060 & My surrounding residents don't visit each other at their lives. & .651 \\
VAR00006 & Being excluded by the original urban residents after changing permanent resident registration. & .617 \\
VAR00015 & The change of timetable impacts my life and work severely. & .616 \\
VAR00020 & I don't like the shopping environment and style of cities. & .579 \\
VAR00005 & I am always despised by original urban residents after changing permanent resident registration. & .573 \\
VAR00021 & I am always hesitate when shopping in cities. & .558 \\
Eigenvalue & 5.31 & \\
Cumulative $\%$ & 12.35 & \\
\hline
\end{tabular}

Table 3. Interpersonal adaptation

\begin{tabular}{lll}
\hline Variable & Item & Factor Loading \\
\hline VAR00049 & I am happy to associate with the original urban residents. & .675 \\
VAR00059 & I often help the residents around me. & .643 \\
VAR00053 & I trust the residents around me very much. & .635 \\
VAR00052 & The original urban residents trust me very much. & .633 \\
VAR00050 & I get along very well with the original urban residents. & .609 \\
VAR00058 & The original urban residents often help me. & .595 \\
VAR00055 & The original urban residents can tolerate my faults. & .564 \\
Eigenvalue & 3.66 & \\
Cumulative $\%$ & 8.51 & \\
\hline
\end{tabular}


Table 4. Values for adaptation

\begin{tabular}{lll}
\hline Variable & Item & Factor Loading \\
\hline VAR00041 & I don't admire the style of celebrating a festival of urban residents. & .734 \\
VAR00040 & I can't bear the recreational activities of urban residents. & .734 \\
VAR00043 & We think in different ways compared with urban residents. & .687 \\
VAR00045 & I am difficult to follow up with the way of thinking of urban residents. & .684 \\
VAR00044 & Our way of thinking makes urban residents astonishing. & .680 \\
VAR00046 & I am incompatible with the entertainment consumption of urban residents. & .651 \\
VAR00038 & I am difficult to accept the views of marriage of urban residents. & .625 \\
VAR00037 & I am hard to adapt to the attitudes towards money of urban residents. & .623 \\
VAR00039 & I can't adapt to the views of human feelings of urban residents. & .503 \\
Eigenvalue & 4.88 & \\
Cumulative $\%$ & 11.34 & \\
\hline
\end{tabular}

Table 5. Psychological adaptation

\begin{tabular}{lll}
\hline Variable & Item & Factor Loading \\
\hline VAR00011 & I am confident in the future of my urban life. & .789 \\
VAR00012 & I believe that living in the city will be better than living in the original place of residence. & .685 \\
VAR00010 & I'm proud of being an urban resident. & .668 \\
VAR00029 & I'm quite pleased with my work environment. & .533 \\
VAR00033 & I am competent in my work in the city. & .481 \\
VAR00007 & I live in the city is my hometown. & .434 \\
VAR00001 & I'm an urban resident after changing permanent resident registration. & .426 \\
VAR00004 & City residents can treat me equally after changing permanent resident registration. & .415 \\
Eigenvalue & 3.13 & \\
Cumulative $\%$ & 7.27 & \\
\hline
\end{tabular}

\subsection{Item Analysis}

The correlation analysis between the score of each item and the total score of the scale was performed, and the Pearson correlation coefficients were regarded as indexes of item discrimination. Results of the Pearson correlation coefficients are shown in Table 6.

As is shown in Table 6, all correlation coefficients( $r$ ) are from 0.145 to 0.632 , and they are all significantly correlated $(\mathrm{p}<0.001)$. So the scale of new citizens' adaptation to urban society has ideal project degree of differentiation.

Table 6. Pearson correlation coefficients

\begin{tabular}{|c|c|c|c|c|c|c|c|c|c|}
\hline W A & & $\mathbf{E} \mathbf{A}$ & & I A & & $\mathbf{V A}$ & & $\mathbf{P A}$ & \\
\hline Item & coefficient & Item & coefficient & Item & coefficient & Item & coefficient & Item & coefficient \\
\hline 31 & $.449 * *$ & 23 & $.320 * *$ & 49 & $.429 * *$ & 41 & $.592 * *$ & 11 & $.548 * *$ \\
\hline 28 & $.427 * *$ & 16 & $.515^{* *}$ & 59 & $.232 * *$ & 40 & $.632 * *$ & 12 & $.352 * *$ \\
\hline 30 & $.560 * *$ & 22 & $.416 * *$ & 53 & $.342 * *$ & 43 & $.519 * *$ & 10 & $.346 * *$ \\
\hline 26 & $.560 * *$ & 24 & $.550 * *$ & 52 & $.306 * *$ & 45 & $.596^{* *}$ & 29 & $.365 * *$ \\
\hline 35 & $.438 * *$ & 60 & $.461 * *$ & 50 & $.350 * *$ & 44 & $.523 * *$ & 33 & $.346 * *$ \\
\hline 13 & $.585^{* *}$ & 15 & $.515^{* *}$ & 55 & $.145^{* *}$ & 38 & $.593 * *$ & 1 & $.367 * *$ \\
\hline 19 & $.392 * *$ & 20 & $.569 * *$ & & & 37 & $.598 * *$ & 4 & $.400 * *$ \\
\hline \multirow[t]{2}{*}{14} & $.585^{* *}$ & 5 & $.435 * *$ & & & 39 & $.571 * *$ & & \\
\hline & & 21 & $.502 * *$ & & & & & & \\
\hline
\end{tabular}

Two-tailed value, ${ }^{*} \mathrm{p}<.05$. Two-tailed $\mathrm{p}$ value, ${ }^{* *} \mathrm{p}<.001$. Note: PA: Psychological Adaptation; EA: Environmental adaptation; VA: Values for adaptation; IA: Interpersonal adaptation; WA: Work Adaptation.

\subsection{Analysis of Reliability and Validity}

\subsubsection{Reliability Analysis}

Table 7 shows the Cronbach's $\alpha$ of the whole scale is 0.908 , and the Cronbach's $\alpha$ of each subscale is above 0.70 , which indicates that the scales have good reliability.
Table 7. Reliability coefficients of scale

\begin{tabular}{ll}
\hline Factor & $\boldsymbol{\alpha}$ \\
\hline Work adaptation & .890 \\
Environment adaptability & .845 \\
Interpersonal adaptation & .785 \\
Values for adaptation & .791 \\
Psychological adaptation & .752 \\
Social adaptation & .908 \\
\hline
\end{tabular}




\subsubsection{Validity Analysis}

1- Content validity: The research group goes through a large number of related documents of home and abroad, having a classified analysis of 187 open questionnaires and 76 in-depth interview records. Meanwhile, during the process of the item compile and modification, the relevant experts have repeatedly investigated it to make the items can reflect the true state of new citizens' adaption to urban society, which ensures the scale has a high content validity.

2- Structure validity: Correlation coefficients of social adaptation subscales of new citizens are $0.021 \sim 0.604$, which is a low and medium correlation; Urban social adaptation subscales and total scale correlation coefficients are $0.462 \sim 0.778$, which is a medium and high degree of correlation. Therefore, they indicate that urban social adaptation has a high validity. As is shown in Table 8.

According to the structure of scale, it's implemented a confirmatory factor analysis for 580 sample data. LISREL8.70 was employed during confirmatory factor analysis testing. Previous studies concluded that there is indicative of acceptable model fit: $\mathrm{X} 2 / \mathrm{df}<3$ ( 5 for looser value), $\quad$ RMSEA $<0.08, \quad \mathrm{NFI}>0.9, \quad \mathrm{NNFI}>0.9, \quad \mathrm{RFI}>0.9$, CFI $>0.9$ (Wu 2009) [24]. The results of Confirmatory Factor Analysis show that $\mathrm{X} 2=3211.80, \mathrm{X} 2 / \mathrm{df}=3.78, \mathrm{RMSEA}=$ 0.073, $\quad \mathrm{NFI}=0.89, \quad \mathrm{NNFI}=0.9, \quad \mathrm{RFI}=0.89, \quad \mathrm{CFI}=0.92$. Therefore, it was considered an acceptable model fit. Result of Confirmatory Factor Analysis is shown in Figure 1.

Table 8. Construct validity of scale

\begin{tabular}{lllll}
\hline scale & W A & E A & I A & V A \\
\hline E A & $.583^{* *}$ & & & \\
I A & $.091^{*}$ & .018 & $.153^{* *}$ & $.527 * *$ \\
C A & $.604^{* *}$ & $.280^{* *}$ & $.402^{* *}$ & $.246^{* *}$ \\
P A & $.332^{* *}$ & $.734^{* *}$ & $.458^{* *}$ & $.776^{* *}$ \\
S A & $.777^{* *}$ & P & $.636^{* *}$ \\
\hline
\end{tabular}

Two-tailed value, ${ }^{*} \mathrm{p}<.05$. Two-tailed $\mathrm{p}$ value, ${ }^{* *} \mathrm{p}<.001$. Note: PA: Psychological Adaptation; EA: Environmental adaptation; VA: Values for adaptation; IA: Interpersonal adaptation; WA: Work Adaptation.

Note: PA: Psychological Adaptation; EA: Environmental adaptation; VA: Values for adaptation; IA: Interpersonal adaptation; WA: Work Adaptation.

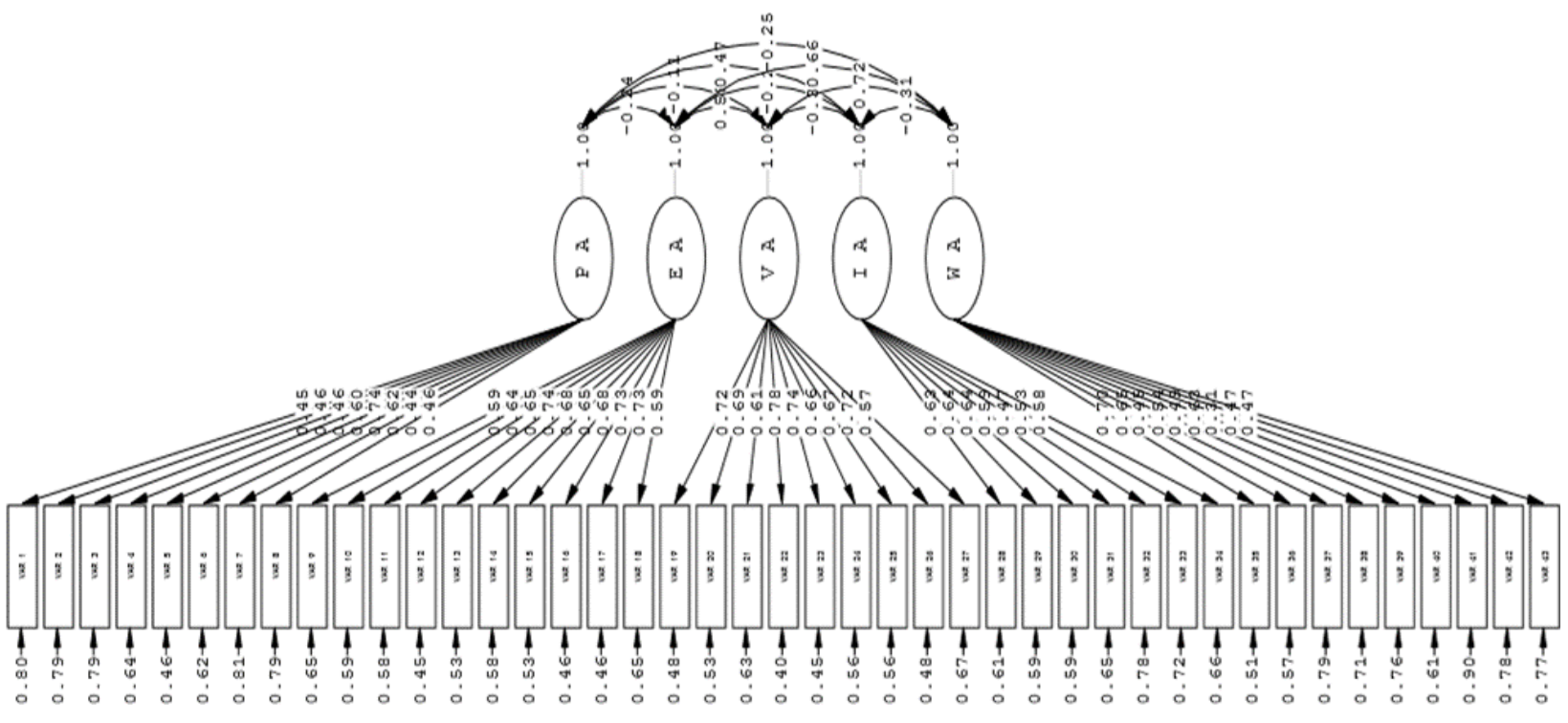

Figure 1. The Standardized path graph of scale

\section{Discussion}

New citizen is a unique group who is characterized by autonomy of rural migrant workers and persistence of long-term migrant. When putting forward the concept of new citizens' adaptation to urban society and drawing up the formal scale, we go through the related documents about sociology and psychology of home and abroad ,classify and analyse lots of opened questionnaires and in-depth interview records and focus on the studies on social adaptation of immigrant, college students, recruit and rural migrant workers The results of exploratory factor analysis shows the new citizens' adaptation to urban society includes five factors: work adaptation, environment adaptation, interpersonal adaptation, values for adaptation, and psychological adaptation. Confirmatory factor analysis shows the rationality and effectiveness of the structure of the scale. Through reliability and item analysis, the results suggested the Cronbach's $\alpha(\alpha=0.903)$ and indexes of item discrimination ( $r=0.145 \sim 0.632)$ of the scale is good.

As a special group of contemporary China, the combination of external environment and internal personal factors that the new citizens are faced with is considerable independent. Using the scale that only for social adaptation 
of the general population to measure the new citizens' adaptation to urban society, it is difficult to obtain a high validity. Moreover, related researches at home and abroad on new citizens' adaptation to urban society are mostly from the perspective of the sociology, which needs a further definition and research from the angle of the psychology.

Compared with the previous studies of new citizens' adaptation to urban society, the research group merge the living conditions adaptation into environment adaptation, which is in line with the traditional concept that makes family as the center of the living environment and is consistent with the study of Feng [22]. It is commonly accepted by academic circle that psychological adaptation is the final phase and the criterion for adaptation of new immigration [19-22].Therefore, psychological adaptation is an absolutely necessary factor of new citizens 'adaptation to urban society. Meanwhile, based on people are likely to be identified with the collective in Eastern context, and the needs of reality that the average score of 580 participants is only 2.005 ( $<2$ as the standard for unadaptable), it is reasonable to establish values for adaptation as a separate dimension.

Based on the current scenario, the New Citizen Unban Social Adaptation Scale has several characteristics: Firstly, developing of the Scale is built on the operating definition of unban social adaptation and the theoretical constructs of five dimensions, which is just and impartial. Secondly, taking full account of Confucian culture and the thought of they do not care to migrate from their native land have large influence of new citizens in the process of scale developing. Therefore, the scale is linked to Chinese cultural background. Thirdly, the scale is the first comprehensive scale which targets the unique group of new citizen under the background of the household registration system reform in china.

The study investigated new citizens' adaptation to urban society and developed measurement tool of high reliability and validity, but there are many follow-up studies to be done. On one hand, it needs a large-scale empirical to investigate its empirical validity so that it further ensures the validity of the scale. On the other hand, since the corresponding limits, the number of samples used in this study is relatively less and it only includes new citizen sample of Chongqing, which lead to the scale has some limitations in sample selection. So, conducting surveys and collecting new citizens' samples which have nationally representativeness to better ensure its external validity.

\section{Conclusion}

Empirical studies have initially obtained the following conclusions: (1) The new citizen urban social adaptation scale has five dimensions: work adaptation, environment adaptation, interpersonal adaptation, values for adaptation, psychological adaptation, which includes 43 items in total. (2) The test results of the reliability and validity show that the Scale has reasonable reliability, validity and model fit, and it is qualified to be a measure tool of new citizens' adaptation to urban society.

\section{Acknowledgements}

The authors would like to thank the reviewers for their helpful comments and suggestions on this research. This work was substantially supported by a grant from the National Social Science Found Project (11XSH019), and Key research project from key research base of Chongqing humanities and social sciences: "Status and Strategy of New Citizens' Adaption to Urban Society in Chongqing" (10SKB22).

\section{References}

[1] Engel, G. L. (1977). The need for a new medical model: a challenge for biomedicine. Science, 196, 129-136.

[2] Bao, L.-f. (2006).Urban adaptation and rural migrants crime. China Agricultural University Journal of Sciences Edition, 24,104-115.

[3] Chen, B. B. (2014). Rural-to-Urban Migrant Children's Behaviors and Adaptation within Migration Social Contexts in China. In Global Perspectives on Well-Being in Immigrant Families (pp. 75-94). Springer New York.

[4] Li, Y.-h., \& Wang, Y.-z. (2012). Review of researches on Social Adaptation Problem. Health Medicine Research and Practice, 9, 75-78.

[5] Li, M.-h. (2000).An overview of the western Theories of International Migration in the $20^{\text {th }}$ century. Journal of XiaMen University(Arts \&Social Sciences), 144,12-18.

[6] Harrison, P. L. (1987). Research with adaptive behavior scales. The Journal of Special Education, 21, 37-68.

[7] Li, Y.-h., \&W, Y.-z. (2012). Review of researches on Social Adaptation Problem. Health Medicine Research and Practice, 9, 75-78.

[8] Wang, Y.-1., \& Lin, C.-d. (2005).The development of the Children' Social-life Adjustment Scale and its application. Psychological Development and Education, 1,109-114.

[9] Yang, Y.-p. (2007).The development of the Middle School Student's Social Adaptation Scale. Psychological Development and Education, 4,108-114.

[10] Fang, X.-y., Wo, J-z., \&Lin, X.-y. (2005). Development of Chinese College Student Adjustment Scale. Studies of Psychology and Behavior, 3, 95-101.

[11] Xiao, L.-j., Miao, D-1., Wang, J-s., Huang-Fu, E., Luo, Z-x., \& Chen, J. (2004). Preliminary Scale of Recruits' Adjustability to Military Life. J Fourth Mil Med Univ, 25, 2027-2030.

[12] Yang, Y.-p., \& Jin, Y. (2006).A review of the research on social adaptation. Psychological Science, 29, 1171-1173.

[13] Paris, W., \& White-Williams, C. (2005). Social adaptation after cardiothoracic transplantation: a review of the literature. Journal of Cardiovascular Nursing, 20, S67-S73. 
[14] Zhu, Z-x. (1989).The Comprehensive Dictionary of Psycho logy, BJ: Beijing Normal University Publishing Group.

[15] Lu, X.-f. (2003).The construction and standardization of "College Student Adaptability Inventory". Central China Normal University.

[16] Jia, X.-b. (2001)The natural and mechanism of mental adaptation. Journal of Tianjin Normal University (Social Science), 154, 19-23.

[17] Neto, F. (2002). Social adaptation difficulties of adolescents with immigrant backgrounds. Social Behavior and Personality: an international journal, 30, 335-345.

[18] Berry, J. W., Phinney, J. S., Sam, D. L., \& Vedder, P. (2006). Immigrant youth: Acculturation, identity, and adaptation. Applied psychology, 55, 303-332.

[19] Jasinskaja - Lahti, I. (2008).Long - term immigrant adaptation: Eight - year follow - up study among immigrants from Russia and Estonia living in Finland. International Journal of Psychology, 43, 6-18.
[20] Tao, S. (2000). Adolescents' Adjustment during transition to University: From the perspective of Life-span development. Journal of Beijing Normal University (Social Science), 158, 81-87.

[21] Tian, K. (1995). Investigation and reflection of urban adaptation of Migrant Workers. Social Science Research, 5, 90-95.

[22] Feng, X-t. (2004). "Falling to the Ground and taking Roots"? _ Adaptation of the rural migrants of the Three Gorges. Sociological Research, 5, 19-27.

[23] Wang, Y.-f., Qin, Q-w., \&Pan, X.-f. (2013).The impact of active development tendency on the urban life adaptation for New Citizens: The mediating effect of social support. Chinese Journal of Applied Psychology, 19, 80-89.

[24] Wu, M.-1. (Eds.) (2009). Structural Equation Modeling, CC: Chongqing University Press. 